\title{
Reproducibility of a silicone-based test food to masticatory performance evaluation by different sieve methods
}

\author{
Alfonso SÁNCHEZ-AYALA(a) \\ Larissa Soares Reis VILANOVA ${ }^{(b)}$ \\ Marina Abrantes COSTA ${ }^{(c)}$ \\ Arcelino FARIAS-NETO(c)
}

\footnotetext{
(a)Department of Dentistry, Dental School, Universidade Estadual de Ponta Grossa UEPG, Ponta Grossa, PR, Brazil.

(b) Department of Prosthodontics and Periodontology, Piracicaba Dental School, Universidade Estadual de Campinas UNICAMP, Piracicaba, SP, Brazil.

(c)Department of Prosthodontics, Dental School, Universidade Potiguar - UnP, Natal, RN, Brazil.
}

Declaration of Interests: The authors certify that they have no commercial or associative interest that represents a conflict of interest in connection with the manuscript.

Corresponding author

Alfonso Sánchez-Ayala

E-mail:snzcd@uepg.br

DOI: 10.1590/1807-3107BOR-2014.vol28.0004 Epub Jun 02, 2014

Submitted: Aug 31, 2013

Accepted for publication: Dec 16, 2013

Last revision: Jan 13, 2014

\begin{abstract}
The aim of this study was to evaluate the reproducibility of the condensation silicone Optosil Comfort ${ }^{\circledR}$ as an artificial test food for masticatory performance evaluation. Twenty dentate subjects with mean age of $23.3 \pm 0.7$ years were selected. Masticatory performance was evaluated using the simple (MPI), the double (IME) and the multiple sieve methods. Trials were carried out five times by three examiners: three times by the first, and once by the second and third examiners. Friedman's test was used to find the differences among time trials. Reproducibility was determined by the intra-class correlation (ICC) test $(\alpha=0.05)$. No differences among time trials were found, except for MPI$4 \mathrm{~mm}(p=0.022)$ from the first examiner results. The intra-examiner reproducibility (ICC) of almost all data was high (ICC $\geq 0.92, p<0.001$ ), being moderate only for MPI-0.50 mm (ICC $=0.89, p<0.001)$. The interexaminer reproducibility was high (ICC $>0.93, p<0.001$ ) for all results. For the multiple sieve method, the average mean of absolute difference from repeated measurements were lower than $1 \mathrm{~mm}$. This trend was observed only from MPI-0.50 to MPI-1.4 for the single sieve method, and from IME-0.71/0.50 to IME-1.40/1.00 for the double sieve method. The results suggest that regardless of the method used, the reproducibility of Optosil Comfort ${ }^{\circledR}$ is high.
\end{abstract}

Keywords: Mastication; Reproducibility of Results; Dental Occlusion.

\section{Introduction}

The masticatory performance (MP) test describes masticatory function objectively as the degree of test food comminution achieved by a certain number of chewing cycles. ${ }^{1}$ MP can be affected by occlusal status, skeletal features, mandibular kinematics, and bite force. ${ }^{1,2,3,4,5}$ In turn, it can influence nutrient intake, body mass index (BMI), ${ }^{5}$ gastric health, ${ }^{6}$ glucose metabolism, ${ }^{7}$ and heart rate. ${ }^{8}$

The multiple sieve method of MP assessment involves calculation of the median comminuted particle size $\left(X_{50}\right)$ by grouping cumulative weight percentage data from passes through sieves of decreasing size. ${ }^{1}$ This estimation includes determination of the broadness variable $(b)$, which describes the spread of particle size distribution. ${ }^{2}$ Other distribution percentiles, such as $X_{80}$ and $X_{20}$, can also be analyzed to determine the proportion of extreme size values. ${ }^{9}$ The single ${ }^{3}$ and double ${ }^{10}$ sieve methods are simpler, but arbitrary, clinical alternatives that quantify cumu- 
lative and retained weight percentages from passes through one and two sieves, respectively. However, these methods may not be reliable if the sieve diameter does not closely approximate the degree of sample comminution. ${ }^{1}$

The test food employed in MP assessment should not be sticky, difficult to chew, or affected by saliva, and it must remain stable during storage at room temperature. ${ }^{11}$ The use of natural test foods, such as oilseed, has been discontinued due to their high solubility during mastication, instability over time, and difficulty of homogenizing their fracture forces and deformation throughout their structures. ${ }^{11}$ The Optosil $^{\circledR}$ (Heraeus Kulzer, Hanau, Germany) family of artificial (polydimethylsiloxane) test foods is considered to be suitable, ${ }^{1,2,4,5,6,9,10,11,12,13,14,15}$ as it has produced results with validated correlations with other masticatory factors, such as the number of occlusal pairs and bite force. ${ }^{3,4,5,6}$ However, few studies have properly evaluated the reproducibility of results obtained with Optosil ${ }^{\circledR}$ products [Optosil ${ }^{\circledR} 1980,9,12$ Optosil P Plus ${ }^{\circledast 4}$, and Optosil Comfort $\left.{ }^{\circledR 1,14,15}(\mathrm{OC})\right]$. Interestingly, the use of 5.6-mm Optosil ${ }^{\circledR}$ cubes $^{4,9,12}$ yielded a lower $X_{50}$ value than that obtained with $\mathrm{OC}^{13,14,15}$ in dentate subjects. Moreover, studies of OC have been conducted with larger samples than have those of previous versions of this material.

As test food confection and masticatory tests are time consuming and require specific laboratory equipment, ${ }^{1}$ evaluation of a small sample may be inevitable, but it affects the reliability of estimates of random error. Unpredictably, random error leads to over- or underestimation of the true value of a variable, and may impact the accuracy of differences observed between groups. ${ }^{16}$ Thus, the aim of this study was to evaluate the reproducibility of MP test results obtained with OC using the single, double, and multiple sieve methods in dentate subjects.

\section{Methodology Participants}

The study sample comprised 20 randomly selected dental students ( 5 men, 15 women) with a mean age of $23.3 \pm 0.7$ years. Inclusion criteria were good general health, normal BMI $\left(18.5-24.9 \mathrm{~kg} / \mathrm{m}^{2}\right)$, presence of all natural teeth (without consideration of third molars), and normocclusion. Dietary regimes, lactation or pregnancy, neuromuscular problems, xerostomy (visually evaluated), orofacial or dental pain, periodontal disease, occlusal caries, severe dental wear, and orthodontic treatment in the last 3 years served as exclusion criteria. Gender was not balanced in the sample because no influence of this variable has been observed in dentate subjects. ${ }^{1}$

The required sample size $(n=11.9)$ was calculated with a two-tailed paired Student's $t$-test $(\alpha=0.05)$ using the mean and standard deviation $(0.29 \pm 0.32)$ of $X_{50}$ differences obtained in a pilot study in which 10 subjects selected according to similar criteria performed two MP time trials weekly. The pilot study also proved the absence of a learning curve during MP test execution, as repeated-measures analysis of variance revealed no difference in successive measures obtained in nine time trials $(p=0.869)$. The Ethics Committee of Universidade Potiguar approved the research protocol (no. 189.770) and all participants provided written informed consent.

\section{MP testing}

OC was manipulated according to the manufacturer's instructions to produce cubes with 5.6-mm edges in metal molds. After the material had set, the cubes were stored in an oven at $60^{\circ} \mathrm{C}$ for $16 \mathrm{~h}$ to ensure complete polymerization.

Participants executed three MP trials administered in the morning by the same examiner at 1-week intervals, and one trial each administered by two additional examiners. Each participant was instructed to chew a portion of 17 cubes ( $3.4 \mathrm{~g}$ ) in a habitual manner. ${ }^{13}$ Although most patients are not familiar with the test food or details of proper test execution, participants received no pre-test training to enable assessment of the true reproducibility of results. No feedback control was used to avoid disequilibrium in the conscious/unconscious nature of the masticatory process and consequent oscillations in bite force and chewing rate. ${ }^{2}$ After 20 chewing cycles, counted by the examiner, participants expectorated the particles onto a paper filter placed over a beaker. They were asked to rinse the mouth with $200 \mathrm{~mL}$ water to completely cleanse the oral cavity. The masticated 
particles were stored in an oven at $80^{\circ} \mathrm{C}$ for $25 \mathrm{~min}$. The material was then passed through a stack of up to 10 sieves with apertures ranging from $0.50 \mathrm{~mm}$ to $5.60 \mathrm{~mm}$ in a sieving machine (Bertel Indústria Metalúrgica Ltda., Caieiras, Brazil) for $20 \mathrm{~min}$. The particles retained on each sieve were weighed on a 0.001-g analytical balance (Mark 2060; Bel Engineering, Lombardy, Italy). ${ }^{14}$

\section{Data processing}

MP was described by the calculation of $X_{50}$ values using the nonlinear regression equation $Q_{W}{ }^{-}(X)=1-2^{-\left(X / X_{50}\right)^{b}}$, where $Q_{W}{ }^{-}$is the cumulative weight percentage of particles smaller than $X$ or passing through a certain sieve aperture, $X_{50}$ is the aperture of a theoretical sieve through which $50 \%$ of the weight can pass, and $b$ is the broadness variable. ${ }^{6}$ $Q_{W}{ }^{-}$data obtained with the multiple sieve method were used to determine the real weight percentage of comminuted test food passing through each sieve, defined as the mastication performance index (MPI). ${ }^{1}$ To obtain double-sieve data, the index of masticatory efficiency (IME) was calculated using the empirical formula IME $=100[1-(X+Y) /(2 T-X)]$, where $X$ and $Y$ are the weights of particles retained in the first (coarse fraction) and second (medium fraction) sieves, respectively; and $T$ is the total weight of masticated particles..$^{10}$ This calculation used data obtained by multiple sieving, grouped into coarse and medium fractions.

\section{Statistical analysis}

Data were analyzed with SPSS ${ }^{\circledast}$ software (ver. 20; IBM, Armonk, USA) using two-tailed tests and a 5\% significance level. Mean $X_{50}, b, \mathrm{MPI}$, and IME values were calculated for each subject. The $X_{20} / X_{80}$ ratio obtained with the multiple sieve method was also used to indicate the degree of variation in particle size within the distribution. Assumptions of normality and homogeneity of variance explored with the Shapiro-Wilk and Levene tests, respectively, were not met. Friedman's test and non-parametric multiple comparisons were thus used to estimate differences among time trials.

Reproducibility was examined by calculating intraclass correlation coefficients (ICCs). Intra-examiner reproducibility was assessed by comparing data from the three MP trials administered by the same examiner. To determine inter-examiner reproducibility, results from the third time trial performed by the first examiner were compared with data from the second and third examiners. The method of moments estimator (MME) $\sigma_{r}=\left[\sqrt{\left.\sum_{i=1}^{n}\left(d_{i}-\bar{d}\right)^{2}\right] / 2(n-1)}\right.$ was used to evaluate the accuracy of estimates of random $(\sigma)$ and "true" (standard deviation) error, where $d_{i}$ is the difference in measurements from each time trial, $n$ is the number of cases, and $\bar{d}$ is the mean of differences. Ninety-five percent confidence intervals were also calculated. ${ }^{16}$

\section{Results}

Table 1 presents data obtained by examiners using the single (MPI), double (IME), and multiple $\left(X_{50}, b\right.$, $\left.X_{20} / X_{80}\right)$ sieve methods. No difference was observed among the three time trials administered by the first examiner, excepting a larger MPI- $4.00 \mathrm{~mm}$ value in trial 1 than in trial $2(p=0.022)$. No difference was found between data from the first examiner's trial 3 and the single trials administered by the second and third examiners. The intra-examiner reproducibility of all data was high (ICC $\geq 0.92, p<0.001$ ), with the exception of moderate reproducibility for MPI$0.50 \mathrm{~mm}(\mathrm{ICC}=0.89, p<0.001$; Table 2). Inter-examiner reproducibility was high for all three methods (ICC > 0.93, $p<0.001$; Table 3).

Average means of absolute differences (AMADs) among repeated measurements were $<1 \mathrm{~mm}$ for the multiple sieve method. For the single and double sieve methods, AMADs were $<1 \%$ only for MPI0.50-MPI-1.4 mm and IME-0.71/0.50-IME-1.40/1.00 $\mathrm{mm}$, respectively. All multiple sieve variables had random errors $<1 \mathrm{~mm}$, but the single and double sieve methods yielded similar errors only for sieves with smaller apertures (Tables 2 and 3). The largest intra- and inter-examiner random error values were for MPI-5.6 and MPI-4.0 mm, respectively.

\section{Discussion}

The results of MP testing with OC using the three methods investigated were highly reproducible. These findings are similar to those of Olthoff et al., ${ }^{12}$ who reported high intra-examiner reproduc- 


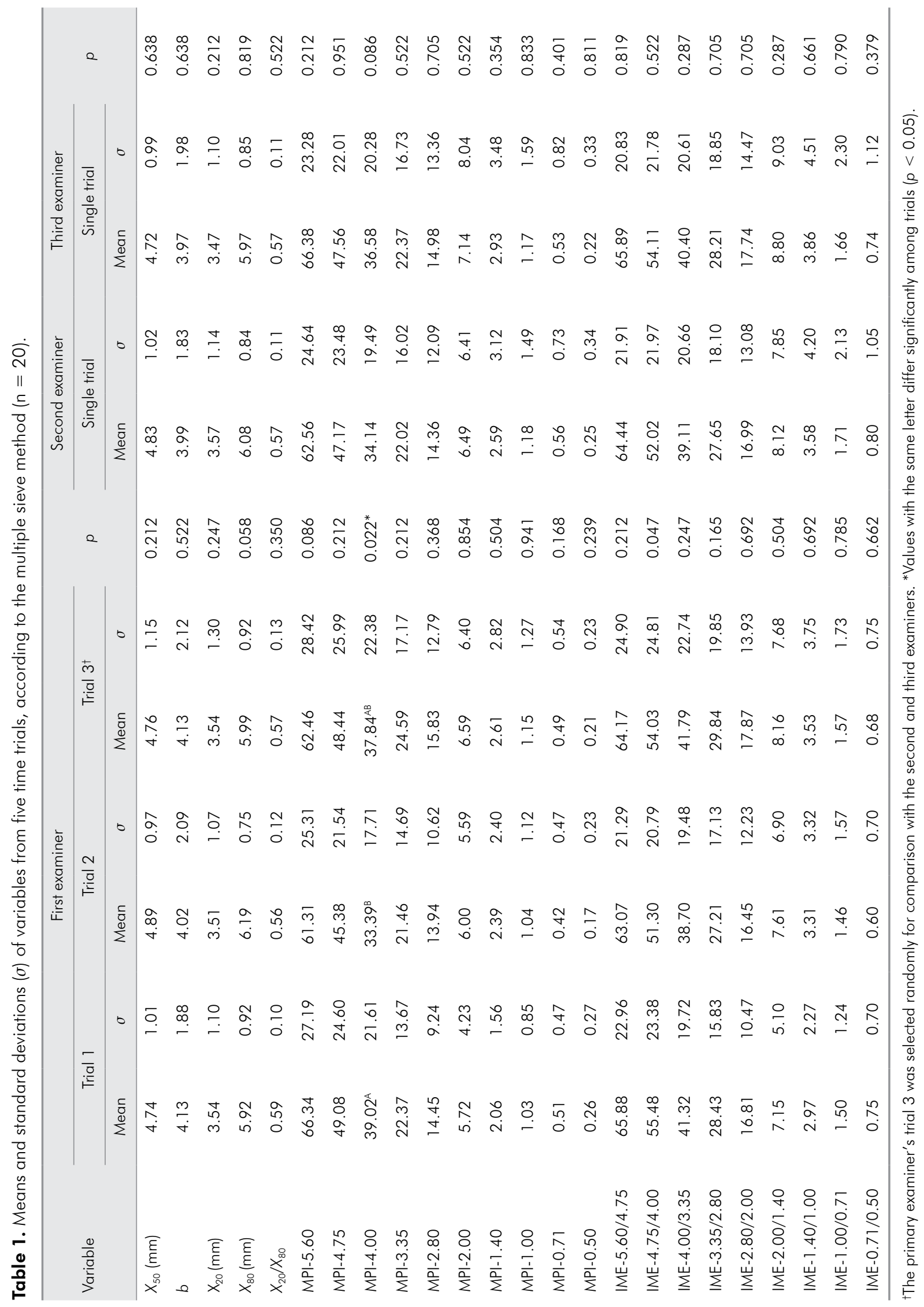


Table 2. Intra-examiner reproducibility and random error in average means of absolute differences, according to the multiple sieve method $(\mathrm{n}=60)$.

\begin{tabular}{|c|c|c|c|c|c|c|c|c|c|}
\hline \multirow{3}{*}{ Variable } & \multirow{3}{*}{$M_{d}$} & \multirow{3}{*}{$\sigma_{\mathrm{d}}$} & \multicolumn{4}{|c|}{ Reproducibility } & \multirow{2}{*}{\multicolumn{3}{|c|}{$\begin{array}{c}\text { Random error } \\
\text { MME }\end{array}$}} \\
\hline & & & \multirow{2}{*}{ ICC } & \multirow{2}{*}{ lower } & \multirow{2}{*}{ upper } & \multirow{2}{*}{$p$} & & & \\
\hline & & & & & & & $\sigma_{\mathrm{r}}$ & lower & upper \\
\hline$X_{50}(\mathrm{~mm})$ & 0.27 & 0.15 & 0.98 & 0.96 & 0.99 & $<0.001$ & 0.11 & 0.06 & 0.15 \\
\hline$b$ & 0.63 & 0.43 & 0.97 & 0.94 & 0.99 & $<0.001$ & 0.30 & 0.16 & 0.42 \\
\hline$X_{20}(\mathrm{~mm})$ & 0.35 & 0.29 & 0.97 & 0.93 & 0.99 & $<0.001$ & 0.20 & 0.11 & 0.29 \\
\hline$X_{80}(\mathrm{~mm})$ & 0.34 & 0.23 & 0.95 & 0.89 & 0.98 & $<0.001$ & 0.16 & 0.09 & 0.23 \\
\hline$X_{20} / X_{80}$ & 0.06 & 0.04 & 0.92 & 0.83 & 0.97 & $<0.001$ & 0.03 & 0.02 & 0.04 \\
\hline MPI-5.60 & 8.53 & 5.06 & 0.97 & 0.94 & 0.99 & $<0.001$ & 3.58 & 1.89 & 5.04 \\
\hline MPI-4.75 & 7.63 & 5.02 & 0.97 & 0.94 & 0.99 & $<0.001$ & 3.55 & 1.87 & 4.99 \\
\hline MPI-4.00 & 7.03 & 3.90 & 0.97 & 0.93 & 0.99 & $<0.001$ & 2.76 & 1.45 & 3.89 \\
\hline MPI-3.35 & 5.59 & 3.91 & 0.96 & 0.91 & 0.98 & $<0.001$ & 2.77 & 1.46 & 3.90 \\
\hline MPI-2.80 & 3.88 & 3.33 & 0.95 & 0.90 & 0.98 & $<0.001$ & 2.36 & 1.24 & 3.32 \\
\hline MPI-2.00 & 1.82 & 2.05 & 0.95 & 0.89 & 0.98 & $<0.001$ & 1.45 & 0.77 & 2.05 \\
\hline MPI- 1.40 & 0.87 & 1.04 & 0.93 & 0.85 & 0.97 & $<0.001$ & 0.73 & 0.39 & 1.03 \\
\hline MPI- 1.00 & 0.36 & 0.43 & 0.94 & 0.88 & 0.98 & $<0.001$ & 0.30 & 0.16 & 0.42 \\
\hline MPI-0.71 & 0.19 & 0.19 & 0.93 & 0.86 & 0.97 & $<0.001$ & 0.14 & 0.07 & 0.19 \\
\hline MPI-0.50 & 0.12 & 0.12 & 0.89 & 0.76 & 0.95 & $<0.001$ & 0.08 & 0.04 & 0.12 \\
\hline IME-5.60/4.75 & 6.08 & 3.38 & 0.98 & 0.96 & 0.99 & $<0.001$ & 2.39 & 1.26 & 3.36 \\
\hline IME-4.75/4.00 & 6.60 & 2.93 & 0.98 & 0.96 & 0.99 & $<0.001$ & 2.07 & 1.09 & 2.92 \\
\hline IME-4.00/3.35 & 6.25 & 3.39 & 0.98 & 0.95 & 0.99 & $<0.001$ & 2.40 & 1.26 & 3.37 \\
\hline IME-3.35/2.80 & 5.62 & 3.28 & 0.97 & 0.94 & 0.99 & $<0.001$ & 2.32 & 1.22 & 3.26 \\
\hline IME-2.80/2.00 & 3.87 & 3.12 & 0.97 & 0.93 & 0.99 & $<0.001$ & 2.20 & 1.16 & 3.10 \\
\hline IME-2.00/1.40 & 2.14 & 2.25 & 0.96 & 0.91 & 0.98 & $<0.001$ & 1.59 & 0.84 & 2.24 \\
\hline IME-1.40/1.00 & 1.12 & 1.27 & 0.94 & 0.88 & 0.97 & $<0.001$ & 0.90 & 0.47 & 1.27 \\
\hline IME-1.00/0.71 & 0.51 & 0.54 & 0.95 & 0.89 & 0.98 & $<0.001$ & 0.38 & 0.20 & 0.54 \\
\hline IME-0.71/0.50 & 0.28 & 0.28 & 0.93 & 0.84 & 0.97 & $<0.001$ & 0.20 & 0.10 & 0.28 \\
\hline
\end{tabular}

ICC: intraclass correlation coefficient; MME: method of moments estimator.

ibility in a study of dentate subjects who executed seven trials each with eight 8 -mm Optosil ${ }^{\circledR}$ cubes. They found no difference in $X_{50}$ values calculated three times per day on 3 different days separated by $\sim$-week intervals. Slagter et al. ${ }^{9}$ found no significant intra-examiner variability in data from seven trials employing 17 5.6-mm Optosil ${ }^{\circledR}$ cubes in dentate subjects and complete denture wearers. Lujan-Climent et al. ${ }^{4}$ reported an ICC of 0.97 and minimum detectable difference of $0.4 \mathrm{~mm}$ for $X_{50}$ values obtained from nine dentate subjects who chewed three-quarters of Optosil P Plus ${ }^{\circledR}$ tablets (5-mm thickness, 20-mm diameter) in five time trials. Using peanuts and the single sieve method, Hatch et al. ${ }^{3}$ obtained an ICC of
0.78 for inter-examiner reliability of MPI- $2 \mathrm{~mm}$ values. Edlund and Lamm ${ }^{10}$ controlled the double sieve method by averaging 10 successive repeated measurements obtained from four subjects performing four trials at 3-day intervals. These researchers and others have concluded that averaging of five $\mathrm{e}^{17}$ or the best four of five ${ }^{10}$ repeated measurements is necessary to adequately determine masticatory efficiency in a study population.

The observed difference in MPI- $4 \mathrm{~mm}$ values between trials 1 and 2 may be related to the susceptibility of the single sieve method to chance fluctuations in the weight of cumulated (passable) or retained particles in sieves. ${ }^{1}$ Not all particles can be comminuted 
Table 3. Inter-examiner reproducibility and random error in average means of absolute differences, according to the multiple sieve method $(n=60)$.

\begin{tabular}{|c|c|c|c|c|c|c|c|c|c|}
\hline \multirow{3}{*}{ Variable } & \multirow{3}{*}{$M_{d}$} & \multirow{3}{*}{$\sigma_{\mathrm{d}}$} & \multicolumn{4}{|c|}{ Reproducibility } & \multirow{2}{*}{\multicolumn{3}{|c|}{$\frac{\text { Random error }}{\text { MME }}$}} \\
\hline & & & \multirow{2}{*}{ ICC } & \multirow{2}{*}{ lower } & \multirow{2}{*}{ upper } & \multirow{2}{*}{$p$} & & & \\
\hline & & & & & & & $\sigma_{\mathrm{r}}$ & lower & upper \\
\hline$X_{50}(\mathrm{~mm})$ & 0.35 & 0.19 & 0.97 & 0.94 & 0.99 & $<0.001$ & 0.13 & 0.07 & 0.19 \\
\hline$b$ & 0.71 & 0.54 & 0.96 & 0.91 & 0.98 & $<0.001$ & 0.38 & 0.20 & 0.53 \\
\hline$X_{20}(\mathrm{~mm})$ & 0.39 & 0.22 & 0.97 & 0.94 & 0.99 & $<0.001$ & 0.16 & 0.08 & 0.22 \\
\hline$X_{80}(\mathrm{~mm})$ & 0.31 & 0.18 & 0.96 & 0.92 & 0.98 & $<0.001$ & 0.13 & 0.07 & 0.18 \\
\hline$X_{20} / X_{80}$ & 0.05 & 0.02 & 0.95 & 0.90 & 0.98 & $<0.001$ & 0.02 & 0.01 & 0.02 \\
\hline MPI-5.60 & 7.75 & 6.39 & 0.97 & 0.93 & 0.99 & $<0.001$ & 4.52 & 2.38 & 6.36 \\
\hline MPI-4.75 & 8.29 & 4.11 & 0.97 & 0.93 & 0.99 & $<0.001$ & 2.91 & 1.53 & 4.09 \\
\hline MPI-4.00 & 8.37 & 6.04 & 0.94 & 0.88 & 0.98 & $<0.001$ & 4.27 & 2.25 & 6.01 \\
\hline MPI-3.35 & 6.14 & 4.80 & 0.95 & 0.90 & 0.98 & $<0.001$ & 3.39 & 1.79 & 4.78 \\
\hline MPI-2.80 & 4.09 & 3.10 & 0.97 & 0.93 & 0.99 & $<0.001$ & 2.19 & 1.15 & 3.08 \\
\hline MPI-2.00 & 2.40 & 1.89 & 0.96 & 0.91 & 0.98 & $<0.001$ & 1.33 & 0.70 & 1.88 \\
\hline MPI- 1.40 & 0.99 & 0.95 & 0.96 & 0.91 & 0.98 & $<0.001$ & 0.67 & 0.35 & 0.94 \\
\hline MPI- 1.00 & 0.43 & 0.35 & 0.97 & 0.94 & 0.99 & $<0.001$ & 0.25 & 0.13 & 0.35 \\
\hline MPI-0.71 & 0.23 & 0.23 & 0.95 & 0.90 & 0.98 & $<0.001$ & 0.16 & 0.08 & 0.23 \\
\hline MPI-0.50 & 0.11 & 0.12 & 0.94 & 0.87 & 0.97 & $<0.001$ & 0.08 & 0.04 & 0.12 \\
\hline IME-5.60/4.75 & 7.06 & 3.76 & 0.97 & 0.94 & 0.99 & $<0.001$ & 2.66 & 1.40 & 3.74 \\
\hline IME-4.75/4.00 & 7.76 & 4.54 & 0.96 & 0.93 & 0.99 & $<0.001$ & 3.21 & 1.69 & 4.52 \\
\hline IME-4.00/3.35 & 7.38 & 5.38 & 0.96 & 0.92 & 0.98 & $<0.001$ & 3.80 & 2.00 & 5.35 \\
\hline IME-3.35/2.80 & 6.29 & 4.67 & 0.96 & 0.93 & 0.99 & $<0.001$ & 3.30 & 1.74 & 4.65 \\
\hline IME-2.80/2.00 & 4.61 & 3.35 & 0.96 & 0.93 & 0.99 & $<0.001$ & 2.37 & 1.25 & 3.33 \\
\hline IME-2.00/1.40 & 2.77 & 2.18 & 0.96 & 0.91 & 0.98 & $<0.001$ & 1.54 & 0.81 & 2.17 \\
\hline IME-1.40/1.00 & 1.30 & 1.02 & 0.97 & 0.93 & 0.99 & $<0.001$ & 0.72 & 0.38 & 1.01 \\
\hline IME-1.00/0.71 & 0.64 & 0.52 & 0.96 & 0.92 & 0.99 & $<0.001$ & 0.37 & 0.19 & 0.52 \\
\hline IME-0.71/0.50 & 0.33 & 0.33 & 0.95 & 0.89 & 0.98 & $<0.001$ & 0.23 & 0.12 & 0.33 \\
\hline
\end{tabular}

ICC: Intraclass correlation coefficient; MME: method of moments estimator. Time trial no. 3 of primary examiner randomly selected for comparison to second and third examiners.

simultaneously during mastication; rather, some particles are selected variably while whole particles remain intact (selection function). ${ }^{9}$ This process depends on the dynamics of the multi-compartment system of the mouth. Comminution occurs in at least one compartment (i.e., on the molars), but not necessarily in other compartments (e.g., interproximal spaces). After a bolus has been swallowed, the residual material in the mouth contains particles $>4 \mathrm{~mm} \cdot{ }^{18}$ The multiple sieve method and, to some extent, the double sieve method enable chance fluctuations in weight to be cancelled out during data analysis because a small weight percentage cumulated or retained in one sieve may be compensated by a large percentage in another. ${ }^{1}$
Despite the high reproducibility demonstrated in this study, the masticatory values obtained with the three methods were lower than those previously reported. ${ }^{4,9,12}$ These differences may be related to anatomical or physiological differences among study populations., ${ }^{3,4}$ They are not likely related to differences in material properties because the Shore A hardness value of $\mathrm{OC}$ is lower than those of previous versions of this material. However, this last presents slightly higher compression strain than OC (www. heraeus-kulzer.com). Body height, tooth size, dental arch length, and habitual diet should be recorded to control for the relative physical capacities of study subjects. In addition, the broadness of and variation in 
particle size distribution represented by $b$ and $X_{20} / X_{80}$ respectively, were higher than previously reported. ${ }^{9}$ However, the relationships between these variables and $X_{50}$ were maintained $(\rho=0.847$ and 0.874 , respectively). Increasing $b$ values correspond to nonlinear regression curves with steeper slopes, and thus to narrower distributions of particle size.,12 Similarly, when $X_{20}$ and $X_{80}$ are close to $X_{50}$, the greatest cumulative weight percentages correspond to near- $X_{50}$ sieves. Thus, the comminuted particles in the present study were of similar sizes (regardless of comminution degree) because mastication was not focused on a few cubes, which depends on synchrony between soft-tissue and mandibular movements. ${ }^{2}$ When more comminution is acheived, the curves are near 0 on the $X$ axis, regardless of the slope.

Although the multiple sieve method includes processing according to simpler methods, the three methods examined here are not necessarily equally suitable for the measurement of MP. ${ }^{1}$ Springate ${ }^{16}$ recommended the use of the MME instead of the con-

\section{References}

1. van der Bilt A, Fontijn-Tekamp FA. Comparison of single and multiple sieve methods for the determination of masticatory performance. Arch Oral Biol. 2004 Mar;49(3):193-8.

2. Sánchez-Ayala A, Farias-Neto A, Campanha NH, Garcia RC. Relationship between chewing rate and masticatory performance. Cranio. 2013 Apr;31(2):118-22.

3. Hatch JP, Shinkai RS, Sakai S, Rugh JD, Paunovich ED. Determinants of masticatory performance in dentate adults. Arch Oral Biol. 2001 Jul;46(7):641-8.

4. Lujan-Climent M, Martinez-Gomis J, Palau S, Ayuso-Montero $\mathrm{R}$, Salsench J, Peraire M. Influence of static and dynamic occlusal characteristics and muscle force on masticatory performance in dentate adults. Eur J Oral Sci. 2008 Jun;116(3):229-36.

5. Sánchez-Ayala A, Campanha NH, Garcia RC. Relationship between body fat and masticatory function. J Prosthodont. 2013 Feb;22(2):120-5.

6. Carretero D, Sánchez-Ayala A, Rodriguez A, Lagravère MO, Gonçalves TM, Garcia RC. Relationship between non-ulcerative functional dyspepsia, occlusal pairs and masticatory performance in partially edentulous elderly persons. Gerodontology. 2011 Dec;28(4):296-301.

7. Yamazaki T, Yamori M, Asai K, Nakano-Araki I, Yamaguchi A, Takahashi K, et al. Mastication and risk for diabetes in a Japanese population: a cross-sectional study. PLoS One. 2013 Jun 5;8(6):e64113. ventionally used Dahlberg's formula to estimate random error, unless the absence of bias among replicate measurements can be demonstrated with certainty. The low error values obtained in this study for multiple sieve data and single and double sieve data for sieves with small apertures may be explained by the cumulated or retained weight fluctuations and differences in selection function described above. Thus, the reproducibility results enable researchers to select a method according to their needs. For larger samples or epidemiological studies requiring only the assessment of differences among populations, simpler methods may be used. For more specific studies requiring explanation of changes in MP, the multiple sieve method may be more appropriate. ${ }^{1}$

\section{Conclusion}

The use of the artificial test food OC for MP measurement using the single, double, and multiple sieve methods generated highly reproducible results in dentate subjects.

8. Takeuchi N, Ekuni D, Tomofuji T, Morita M. Relationship between masticatory performance and heart rate variability: a pilot study. Acta Odontol Scand. 2013 May-Jul;71(3-4):807-12.

9. Slagter AP, Olthoff LW, Steen WH, Bosman F. Comminution of food by complete-denture wearers. J Dent Res. 1992 Feb;71(2):380-6.

10. Edlund J, Lamm CJ. Masticatory efficiency. J Oral Rehabil. 1980 Mar;7(2):123-30.

11. van Der Glas HW, Al-Ibrahim A, Lyons MF. A stable artificial test food suitable for labeling to quantify selection and breakage in subjects with impaired chewing ability. J Texture Stud. 2012 Aug;43(4):287-98.

12. Olthoff LW, van der Bilt A, Bosman F, Kleizen HH. Distribution of particle sizes in food comminuted by human mastication. Arch Oral Biol. 1984;29(11):899-903.

13. Gomes SGF, Del Bel Cury AA, Garcia RCMR. Effect of hyposalivation on mastication and mandibular movements during speech. Braz Oral Res. 2011 Jul-Aug;25(4):351-6.

14. Gonçalves TM, Sanchez-Ayala A, Ambrosano GM, Garcia RC. Female hormonal fluctuation and masticatory function in patients with disc displacement-a case-control study. Int J Prosthodont. 2011 Jul-Aug;24(4):320-7.

15. Eberhard L, Schindler HJ, Hellmann D, Schmitter M, Rammelsberg P, Giannakopoulos NN. Comparison of particle- 
- Reproducibility of a silicone-based test food to masticatory performance evaluation by different sieve methods

size distributions determined by optical scanning and by sieving in the assessment of masticatory performance. J Oral Rehabil. 2012 May;39(5):338-48.

16. Springate SD. The effect of sample size and bias on the reliability of estimates of error: a comparative study of Dahlberg's formula. Eur J Orthod. 2012 Apr;34(2):158-63.
17. Omar SM, McEwen JD, Ogston SA. A test for occlusal function. The value of a masticatory efficiency test in the assessment of occlusal function. Br J Orthod. 1987 Apr;14(2):85-90.

18. Flynn CS, Foster KD, Bronlund JE, Lentle RG, Jones JR, Morgenstern MP. Identification of multiple compartments present during the mastication of solid food. Arch Oral Biol. 2011 Apr;56(4):345-52. 